\title{
Superfluidity and Critical Velocities in Nonequilibrium Bose-Einstein Condensates
}

\author{
Michiel Wouters ${ }^{1}$ and Iacopo Carusotto ${ }^{2}$ \\ ${ }^{1}$ Institute of Theoretical Physics, Ecole Polytechnique Fédérale de Lausanne (EPFL), CH-1015 Lausanne, Switzerland \\ ${ }^{2}$ INO-CNR BEC Center and Dipartimento di Fisica, Università di Trento, I-38123 Povo, Italy \\ (Received 11 December 2009; revised manuscript received 11 March 2010; published 8 July 2010)
}

\begin{abstract}
We theoretically study the superfluidity properties of a nonequilibrium Bose-Einstein condensate of exciton polaritons in a semiconductor microcavity under incoherent pumping. The dynamics of the condensate is described at mean-field level in terms of a generalized Gross-Pitaevskii equation. The drag force on a small moving object and the onset of fringes in the density profile are shown to have a sharp threshold as a function of the velocity; a generalized Landau criterion is developed to explain this behavior in terms of the dispersion of elementary excitations. Metastability of supercurrents in multiply-connected geometries is shown to persist up to higher flow speeds.
\end{abstract}

PACS numbers: 05.70.Ln, 03.75.Kk, 67.90.+z, 71.36.+c

Superfluidity is among the most remarkable consequences of macroscopic quantum coherence in condensed matter systems and manifests itself in a number of fascinating effects $[1,2]$. A unified description of these phenomena is obtained in the framework of the so-called two-fluid hydrodynamics, in which the macroscopic condensate wave function adds up to the standard hydrodynamic variables [3]. The phenomenon of macroscopic coherence is not restricted to systems at (or close to) thermodynamical equilibrium such as liquid Helium, ultracold atomic gases, or superconducting materials, but has been observed also in systems far from thermodynamical equilibrium, whose stationary state is determined by a dynamical balance of driving and losses. Most remarkable examples are lasers and, more recently, Bose-Einstein condensates of magnons in magnetic solids [4] and exciton polaritons in semiconductor microcavities [5-7]. In particular, the issue of superfluidity in this latter system has attracted a significant interest from both the theoretical [8-14] and experimental $[10,15,16]$ points of view.

Recent experiments with resonantly pumped polariton condensates [15] have demonstrated superfluidity as a dramatic reduction in the intensity of resonant Rayleigh scattering, as originally predicted in [8]. The situation is less clear in the case of nonresonant or parametric (OPO) pumping schemes: recent experiments in this latter configuration [16] have observed propagation of polariton bullets without apparent friction, which is in contrast with the predictions of a naïve Landau criterion based on the elementary excitation spectrum predicted in [12]. Another aspect of superfluidity, namely metastability of supercurrent states in multiply-connected geometries was theoretically investigated in $[9,10]$ and experimentally confirmed in [10].

The present Letter reports a comprehensive theoretical investigation of the meaning of superfluidity for nonresonantly pumped polariton condensates [5-7]. Emphasis is given to the novel features that originate from their non- equilibrium character. As condensation in both nonresonant and OPO pumping schemes originates from the same $U(1)$ spontaneous symmetry-breaking mechanism, we expect that our results can provide useful theoretical insight into the superfluidity features observed in [16] under an OPO pumping.

At the mean-field level, the condensate dynamics can be described in terms of the Gross-Pitaevskii equation (GPE) [1], which was recently generalized to nonequilibrium condensates by including the effect of pumping and losses $[13,17]$. This description has been able to explain a number of experimental observations, e.g., the ring-shaped momentum distribution of spatially narrow condensates $[6,18]$, the synchronization transition [19], and the spontaneous appearance of vortices [20]. Nonetheless, the implicit assumption that the pumping mechanism is not frequency selective can lead to unphysical predictions, e.g., that in a spatially homogeneous or ringlike geometry condensation is equally likely to occur in any momentum state. Kinetic calculations [21] have pointed out the significant frequency dependence of the polariton-polariton scattering processes that are responsible for replenishing the condensate. A simplest generalization of the GPE to include a frequency dependence of the pumping has the form:

$$
\begin{aligned}
i \frac{d \psi}{d t}= & \left\{-\frac{\hbar}{2 m} \nabla^{2}+V_{\mathrm{ext}}+g|\psi|^{2}\right. \\
& \left.+\frac{i}{2}\left[P\left(1-\frac{i}{\Omega_{K}} \frac{d}{d t}\right)-\gamma-r|\psi|^{2}\right]\right\} \psi .
\end{aligned}
$$

The amplification efficiency (proportional to the pumping strength $P$ ) decreases to zero a frequency $\Omega_{K}$ above the bottom of the polariton branch. Assuming a linear dependence of amplification on frequency, a temporally local form is maintained in (1). The other terms describing gain saturation $(r)$, losses $(\gamma)$, polariton mass $(m)$, polaritonpolariton interactions $(g)$, external potential $\left(V_{\text {ext }}\right)$ have the same meaning as in $[13,17]$. 
We first consider the evolution of the system starting from an initial state with no condensate $\psi=0$. If the strength of pumping is enough to overcome losses $P>$ $\gamma$, the $\psi=0$ state is dynamically unstable against the creation of a finite condensate amplitude in any of the low-momentum modes for which $\hbar k^{2} / 2 m<\Omega_{K}(1-$ $\gamma / P)$. The rate of this instability is maximum at $k=0$ and decreases for increasing $k$. This fact is in agreement with the experimental observation of condensation naturally occurring around $k=0$ as soon as the sample is sufficiently large and free from disorder [5].

In spite of this natural preference, condensation can be forced to occur in finite momentum state by seeding the system with a short coherent light pulse at the desired $\mathbf{k}_{c}$, which amounts to adding a term of the form $F_{s} \delta(t) e^{i \mathbf{k}_{c} \mathbf{r}}$ to the right-hand side of (1). The efficiency of this procedure to create finite momentum condensates was numerically assessed in [9] and a related configuration was experimentally demonstrated for the OPO pumping scheme in [10]. Well after the seed pulse, the density of the moving condensate tends to its steady-state value:

$$
n_{c}=|\psi|^{2}=\frac{1}{r}\left\{\left[1-\frac{1}{\Omega_{K}}\left(\frac{\hbar k_{c}^{2}}{2 m}+g|\psi|^{2}\right)\right] P-\gamma\right\} .
$$

The dynamical stability of the moving condensate is to be assessed by linearization of the generalized GPE (1) around the stationary solution. Examples of the Bogoliubov dispersion $\omega_{\text {Bog }}(q)$ for moving condensates are shown in Fig. 1. In the leftmost panels (a) and (b), the limit $\Omega_{K} \rightarrow \infty$ of a negligible frequency dependence of pumping is considered. Apart from the global Doppler
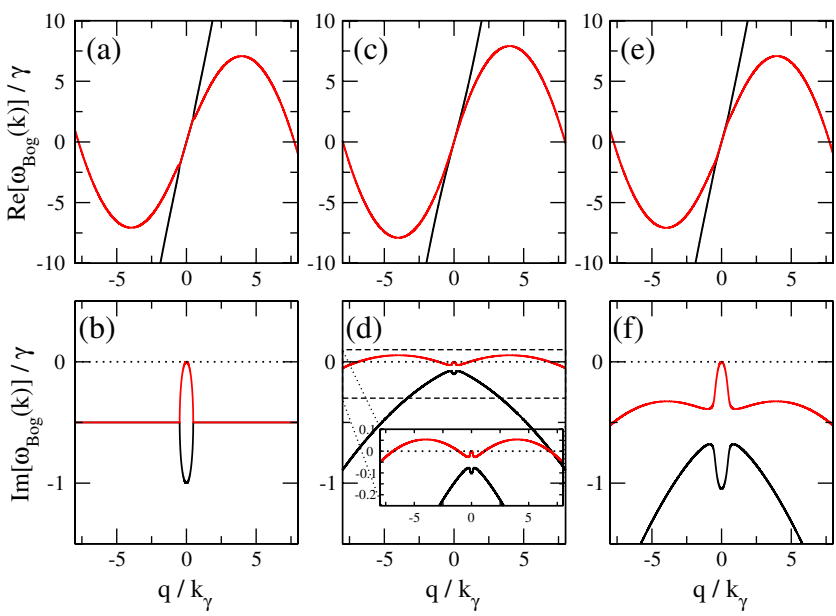

FIG. 1 (color online). Elementary excitation spectrum of a moving, spatially homogeneous, nonequilibrium condensate as described by the linearized generalized GPE around the steadystate solution. Parameters: $k_{c} / k_{\gamma}=4, r / \gamma=1, g / \gamma=1, \Omega_{K} /$ $\gamma=50$, Panels (c),(d) and (e),(f) correspond to different values of the density, $n_{c} g / \gamma=0.1$ [(c),(d)] and $n_{c} g / \gamma=1$ [(e),(f)], respectively. Inset: magnified view of (d). Panels (a),(b): same as panels (e),(f) in the limit of a frequency-independent pumping $\Omega_{K}=\infty$. Momenta are measured in units of $k_{\gamma}=\sqrt{m \gamma / \hbar}$. tilting of the real part due to the finite condensate velocity, the plotted dispersion fully recovers the diffusive character first discussed in [11-13]: a flat dispersion around the condensate wave vector and a negative imaginary part quadratically growing in $q$. In this limit, stable condensates exist for any value of $k_{c}$.

The effect of a frequency-selective pumping is addressed in the other panels where $\Omega_{K}$ has a large, but finite value. The central panels (c) and (d) refer to the case with a very small condensate density, where the real part of the Bogoliubov mode frequency reduces to the single-particle one. On the other hand, the characteristic damping rate of density fluctuations (the gapped mode at $q=0$ ) and of the high-momentum modes is suppressed by a critical slowing down phenomenon [13]. As a result, the imaginary part of the low-energy modes around $q \simeq-k_{c}$ become positive, which signals a dynamical instability. Eventually, this instability leads to the disappearance of the original condensate and the formation of another stable condensate in a lower momentum state.

The right panels (e) and (f) refer to the case of a higher density. In this case, the damping rate of all modes other than the Goldstone one is comparable to the polariton lifetime $\gamma$ and the frequency dependence of pumping is not able to destabilize the moving condensate. The stability domain as a function of the momentum $k_{c}$ and the density $n_{c}$ of the condensate is summarized in Fig. 2: as the density is increased, stable condensates survive up to larger momenta. It is of crucial importance to note that this instability mechanism has no direct counterpart in standard equilibrium superfluids and is effective even in the absence of any defect potential.

In the presence of weak defects, the frictionless flow of equilibrium condensates is limited to flow speeds below the Landau critical velocity $v_{c}=\min _{k}\left\{\operatorname{Re}\left[\omega_{\text {Bog }}^{o}(k)\right] / k\right\}$, where $\omega_{\mathrm{Bog}}^{o}(k)$ is the real part of the Bogoliubov dispersion for a condensate at rest. For dilute systems, $v_{c}$ coincides with the sound velocity $c_{s}=\sqrt{g n_{c} / m}$ [1]. As a consequence of the diffusive character of the Goldstone mode, a naïve application of this Landau criterion to the Bogoliubov dispersion of nonequilibrium condensates an-

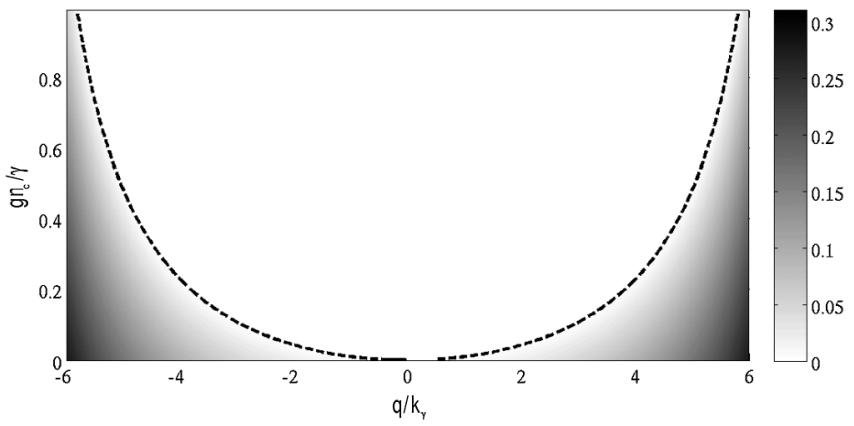

FIG. 2. Growth rate of the maximally unstable mode as a function of condensate momentum $k_{c}$ and density $n_{c}$. The region above the dashed line corresponds to dynamically stable condensates. Same system parameters as in Figs. 1(c) and 1(f). 
ticipated in [11-13] would instead give a vanishing prediction for the critical velocity, $v_{c}=0$ : Bogoliubov waves are emitted in a moving condensate hitting a (weak) defect for any value of the condensate speed.

However, a complete picture of nonequilibrium condensates requires taking into account the nontrivial dynamics of the imaginary part of the Bogoliubov dispersion. Numerical plots of the density perturbation induced by a single weak stationary defect in a (dynamically stable) moving condensate can be calculated from the generalized GPE (1) and are shown in Fig. 3 for different values of the condensate velocity. In contrast to the predictions of the naïve Landau criterion, the induced perturbation closely resembles the one induced in an equilibrium condensate described by the standard GPE [22]: at high speeds [panel (a)], the defect creates a series of parabolic fringes that propagate away from the defect; at low speeds [panel (c)], the propagating fringes are replaced by a localized perturbation in the vicinity of the defect. Remarkably, the characteristic speed at which the fringes disappear is of the order of the equilibrium sound speed $c_{s}=\sqrt{g n_{c} / m}$, but does not correspond to any feature in the Bogoliubov dispersions shown in Fig. 1.

As it was pointed out in a different context in [23], the emission pattern by a localized monochromatic source is better understood in terms of the (complex) wave vector of the emitted wave at the given (real) perturbation frequency rather than in terms of the (complex) resonance frequency of an elementary excitation with a given (real) wave vector. In our case of a static defect, the perturbation applied to the condensate has zero frequency. As one can see in Fig. 4(b), the wave vector $\tilde{k}$ of the corresponding Bogoliubov wave starts having a finite real part only after a branching point: extended oscillations in the density are observed as soon as the real part of $\tilde{k}$ exceeds the imaginary part.

This behavior is reflected in the drag force $F$ exerted by the moving condensate onto the defect as a function of the condensate velocity [Fig. 4(a)]. In terms of the perturbed density profile $n(\mathbf{r})$, the drag force is given by $F=$ $-\int d^{2} \mathbf{r} n(\mathbf{r}) \nabla_{\mathbf{r}} V_{\text {def }}(\mathbf{r})$ [24]. As a consequence of the finite lifetime of the Bogoliubov modes, the drag force has a nonvanishing value at all $v$. Still, it shows a pronounced threshold at a velocity value that closely corresponds to the onset of fringes in the density profile. Comparison of the
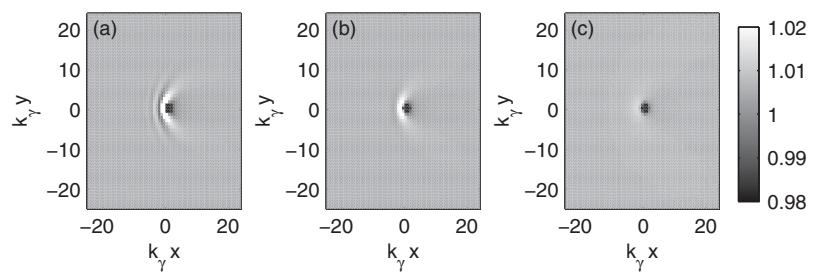

FIG. 3. Density perturbation created in a moving condensate by a stationary weak defect for three values of the condensate velocity $v / c_{s}=1.5,1,0.4$ across the critical value for superfluidity. Parameters: $n_{c} g / \gamma=n_{c} r / \gamma=1, \Omega_{K} / \gamma=50$. curves for different values of the nonequilibrium parameter $\gamma / g n_{c}$ confirms that systems closer to equilibrium display a sharper threshold. The equilibrium limit of [24] is recovered in the limit $\gamma \rightarrow 0$. Constancy of the ratios $P / \gamma$ and $r n_{c} / \gamma$ guarantees a homogeneous scaling of the different loss or amplification terms on the second line of (1). The effect of $\Omega_{K}$ on the drag force is minor.

It is important to note that the present theory is based on the mean-field equation (1) for the condensate wave function: in the steady state that we are considering here, this oscillates at the single frequency $\omega_{c}$. In spite of the different underlying mechanism, the phenomenology that one observes in Fig. 4(a) is however similar to the one of finite temperature equilibrium condensates where a nonvanishing friction appears at all velocities as a consequence of the thermal, noncondensed component. The contribution of the noncondensed component in the present nonequilibrium case will be the matter of future work along the lines of [25].

So far, no experiment has investigated yet the superfluid properties of polariton condensates under nonresonant pumping. Still, it is likely that this generalized form of the Landau criterion provides at least a partial explanation of the recent experimental observation of superfluidity in a OPO regime [16].

Supersonic flows in equilibrium condensates in ringshaped geometries are generally strongly sensitive to the presence of defects: as soon as nodes appear in the condensate wave function, the topological stability of the supercurrent state is broken, which leads to a rapid slow down of the condensate motion. The finite damping rate of excitations in polariton condensates introduces a substantial modification to this picture: the perturbation created by each defect is not able to propagate on long distances, but rather remains localized in space on a length scale inversely proportional to $\operatorname{Im}[\tilde{k}]$. As a result, we expect it is much harder to break the topological stability of the supercurrents.
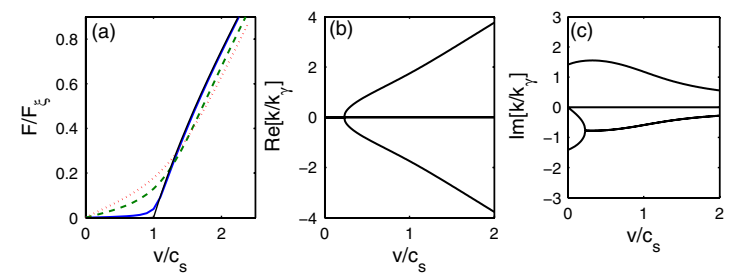

FIG. 4 (color online). Panel (a): Force exerted on a weak stationary defect by a moving condensate as a function of the condensate velocity $v$ for different values of the nonequilibrium parameter $\gamma / g n_{c}=0,0.1,1,2$ (thin black solid line, blue solid line, green dashed line, red dotted line), in units of $F_{\xi}=$ $\hbar^{2} / m \xi^{3}$, where $\xi=\sqrt{\hbar^{2} / m g n_{c}}$. The ratios $P / \gamma$ and $r n_{c} / \gamma$ are kept constant. The other parameters are the same as in Fig. 3. Panels (b),(c): Real and imaginary parts of the complex wave vector $\tilde{k}$ of the zero-frequency Bogoliubov mode as a function of $v$. The wave vector is taken to be parallel to the flow velocity. The parameters correspond to the green curve of panel (a). 

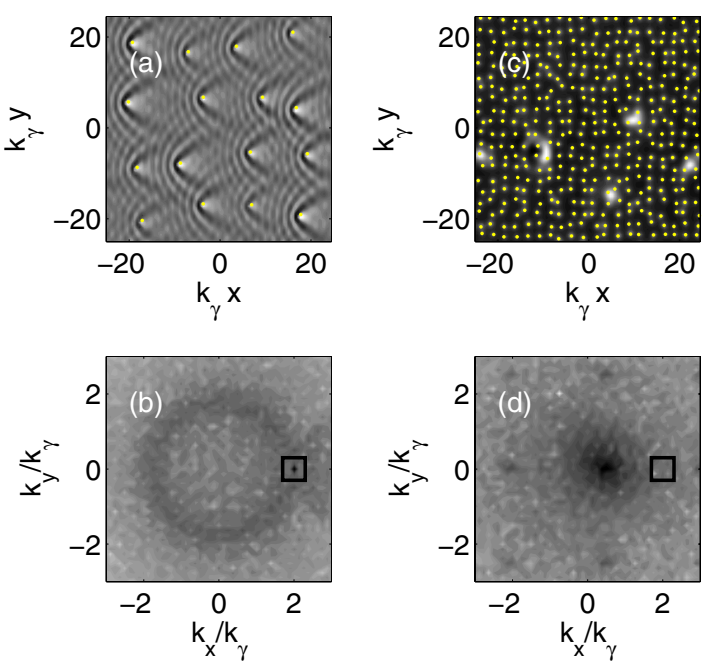

FIG. 5 (color online). Real (linear color scale) and momentum space (logarithmic color scale) densities of a nonequilibrium condensate after a temporal evolution of $\gamma t=300$ for an initial momentum $k / k_{\gamma}=2$ and two different densities of defects. The dots in the real space panels indicate the position of the defects. The square in the momentum space panels indicates the initial momentum of the condensate. Parameters are the same as in Fig. 4, except for $\Omega_{K} / \gamma=10$.

This physical picture is confirmed by numerical simulations of the time evolution under the generalized GPE (1) starting from an initial condition with a supersonic flow. Periodic boundary conditions are assumed. For a low defect density, the supercurrent state is maintained for very long times with no sign of decay. The characteristic Cerenkov-like density patterns in the vicinity of each defect are spatially separated and do not interfere [Fig. 5(a)]. The momentum distribution in $k$ space [Fig. 5(b)] shows the condensate peak right at the initial momentum state and a much fainter resonant Rayleigh scattering ring [8]. The situation is different for a higher density of defects. In this case, the real space density perturbations created by the different defects substantially overlap with each other. As a result, interference effects are more likely to create nodes in the condensate wave function and therefore to trigger dissipation of the supercurrent. The occurrence of such a process is apparent in Fig. 5(d) as a much reduced late-time value of the condensate momentum.

This fact illustrates another important difference with respect to standard, equilibrium condensates: in that case, the dissipation of a supercurrent leads to a significant heating and reduction of the condensate fraction. In the present case, the condensate is transferred to a lower momentum state but maintains its long-range coherence; the momentum broadening that is visible in Fig. 5(d) is related to the localized modulation created by the defects. Even if the presence of some frequency dependence in the pumping is crucial to concentrate the population in lowenergy modes, the final shape of the momentum distribution depends only weakly on its specific form.
In conclusion, we have theoretically investigated the superfluidity properties of nonequilibrium condensates of exciton polaritons. Contrary to previous expectations, superfluidity is shown to be robust against particle loss. Fringes in the density profile are created by a moving defect only above a critical speed; correspondingly, the drag force shows a pronounced thresholdlike behavior. Remarkably, metastability of supercurrents is found to persist even for velocities well above the critical speed.

We are indebted to Vincenzo Savona, Cristiano Ciuti, and Davide Sarchi for continuous enlightening exchanges. Stimulating discussions with E. Cancellieri, J. Keeling, C. Menotti, F. Piazza, D. Sanvitto, and A. Smerzi are acknowledged.

[1] L.P. Pitaevskii and S. Stringari, Bose-Einstein Condensation (Clarendon Press, Oxford, 2003).

[2] A. J. Leggett, Rev. Mod. Phys. 71, S318 (1999).

[3] K. Huang, Statistical Mechanics (John Wiley \& Sons, New York, 1963).

[4] O. Demokritov et al., Nature (London) 443, 430 (2006).

[5] J. Kasprzak et al., Nature (London) 443, 409 (2006).

[6] M. Richard et al., Phys. Rev. Lett. 94, 187401 (2005).

[7] J. Keeling et al., Semicond. Sci. Technol. 22, R1 (2007).

[8] I. Carusotto and C. Ciuti, Phys. Rev. Lett. 93, 166401 (2004).

[9] M. Wouters and V. Savona, Phys. Rev. B 81, 054508 (2010).

[10] D. Sanvitto et al., Nature Phys. 6, 527 (2010).

[11] M. H. Szymańska, J. Keeling, and P. B. Littlewood, Phys. Rev. Lett. 96, 230602 (2006).

[12] M. Wouters and I. Carusotto, Phys. Rev. A 76, 043807 (2007).

[13] M. Wouters and I. Carusotto, Phys. Rev. Lett. 99, 140402 (2007).

[14] I. Carusotto, M. Wouters, and C. Ciuti, Presentation at ICSCE4 (2008), http://www.tcm.phy.cam.ac.uk/BIG/ icsce4/talks/carusotto.pdf; J. Keeling and N. G. Berloff, Nature (London) 457, 273 (2009).

[15] A. Amo et al., Nature Phys. 5, 805 (2009).

[16] A. Amo et al., Nature (London) 457, 291 (2009).

[17] J. Keeling and N. G. Berloff, Phys. Rev. Lett. 100, 250401 (2008).

[18] M. Wouters, I. Carusotto, and C. Ciuti, Phys. Rev. B 77, 115340 (2008)

[19] A. Baas et al., Phys. Rev. Lett. 100, 170401 (2008); M. Wouters, Phys. Rev. B 77, 121302 (2008); P. R. Eastham, Phys. Rev. B 78, 035319(R) (2008).

[20] K. G. Lagoudakis et al., Nature Phys. 4, 706 (2008).

[21] D. Porras, C. Ciuti, J. J. Baumberg, and C. Tejedor, Phys. Rev. B 66, 085304 (2002).

[22] I. Carusotto et al., Phys. Rev. Lett. 97, 260403 (2006).

[23] W. C. Tait, Phys. Rev. B 5, 648 (1972).

[24] G. E. Astrakharchik and L. P. Pitaevskii, Phys. Rev. A 70, 013608 (2004).

[25] M. Wouters and V. Savona, Phys. Rev. B 79, 165302 (2009). 